\title{
TIDAL MODELS IN A NEW ERA OF SATELLITE GRAVIMETRY
}

\author{
R. I. RAY and D. D. ROWLANDS \\ Space Geodesy Branch, NASA Goddard Space Flight Center Greenbelt, USA \\ G. D. EGBERT \\ College of Oceanic \& Atmi spheric Sciences, Oregon State University, Conallis, USA
}

Recerved: ...; Accepted in final form: ...

\begin{abstract}
The high precision gravity measurements to be made by recently launched (and recently approved) satellites place new demands on models of Earth, atmospheric, and oceanic tides. The latter is the most problematic. The ocean tides induce variations in the Earth's geoid by amounts that far exceed the new satellite sensitivities, and tidal models must be used to correct for this. Two methods are used here to determine the standard errors in current ocean tide models. At long wavelengths these errors exceed the sensitivity of the GRACE mission. Tidal errors will not prevent the new satellite missions from improving our knowledge of the geopotential by orders of magnitude, but the errors may well contaminate (IRACE estimates of temporal variations in gravity. Solar tides are especially problematic because of their long alias periods. The satellite data may be used to improve tidal models once a sufficiently ling time series is obtained. Improvements in the long-wavelength components of lunar tides are especially promising.
\end{abstract}

\section{Introduction}

With the recent launches of the CHAMP and GRACE satellite missions and the approval of the GOCE mis sion, we find ourselves entering a new era in satellite gravimetry. The high precisıons and increased sensitivities of these missions place new demands on methodologies and models. This paper examines the role of tides and tide model errors, with special emphasis given to the GRACE low-low satelliteto-satellite tracking mission (Tapley and Reigber, 2000).

Tides have been a key component of space geodesy since the mid-1960's, but their role in satellite gravimetry has been confined primarily (although not exclusively) to determining long-period tidal perturbations in satellite orbits or to estimating certain tidal parameters from such perturbations. Determining long-period orbit perturbations will conlinue to be of utmost importance, in these new missions as well as in other high-precision applications (e.g., Pavlis and lorio, 2002). But in this new era, attention will also turn to two new aspects (cf. Schrama, 1996). (1) The continuous GPS tricking of satellites now allows short-period tidally induced orbit perturbations to be studied, necessitating models with complete sets of spherical harmonic coefficients, not limited to a few select orders. (2) Some of the new data types allow a direct instantaneous measurement of the gravity effects of tides (embedded, of course, in a background of gravity signals from other 
anomaly sources). Unlike long-period orbit perturbations, these direct ${ }^{*}$ effects can be localized near the causat ve masses. Furthermore, the contamination of gravity field measurements (or estinnates) from inadequately modeled tidal masses will be an important concern. In this paper we consider the gravitational effects of tides and examine how errors in tidal models may affect the new satellite gravimetry missions. Space precludes a complete discussion, so we focus on oceanic tides, as opposed to Earth tides or atmospheric tides, because errors in the former are much larger than errors in the lattcr.

\section{Ocean Tides and Gravity}

To understand the extent to which new gravity missions are directly affected by ocean tides, we use a global model to evaluate (1) the tidally induced perturbations in gravity (or the geoid) and (2) the associated signals that can be expected in satellite measurements. Later sections consider corresponding errors in present-day models.

\subsection{Gravity Perturbations Induced by OCEAN Tides}

Suppose the ocean tidal elevations are expanded in normalized complex spherical harmonics $Y_{n}^{m}(\theta, \varphi)$ as

$$
\zeta(\theta, \varphi, t)=\sum_{n, m} z_{n m}(t) Y_{n}^{m}(\theta, \varphi)
$$

The coefficients $z_{n m}(t)$ vary with tidal periodicity. At satellite altitudes the gravitational potential of this tide is given by (e.g., Lambeck, 1988)

$$
U(r, \theta, \varphi, t)=4 \pi G a \rho_{w} \sum_{n, n} \frac{1+k_{n}^{\prime}}{2 n+1}\left(\frac{a}{r}\right)^{n+1} z_{n m}(t) Y_{n}^{m}(\theta, \varphi)
$$

where $\rho_{w}$ is the mean density of seawater (approximately $1035 \mathrm{~kg} \mathrm{~m}^{-3}$ ), $a$ the radius of the Earth, $G$ is the Newtonian constant, and $k_{n}^{\prime}$ are loading Love numbers.

The corresponding induced variations in the geoid are given by (e.g., Schrama, 1996; Wahr, 1998)

$$
\delta N(\theta, \varphi, t)=3\left(\rho_{w} / \rho_{e}\right) \sum_{n, m}\left(\frac{1+k_{n}^{\prime}}{2 n+1}\right) z_{n m}(t) Y_{n}^{m}(\theta, \varphi),
$$

where $\rho_{e}$ is the mean density of the Earth. Figure 1 shows the amplitudes of both $\zeta$ and $\delta N$ for the principal semidiurnal lunar tide $\mathbf{M}_{2}$.

* Here 'direct' is used in contrast with the indirect tidal gravity effect manifested by orbit perturbations. Schrama (1996) prefers 'indirect' for both and reserves 'direct' for the primary astronomical tidal forces of the sun or moon. The latter are exceedingly well known and not discussed in this paper. 

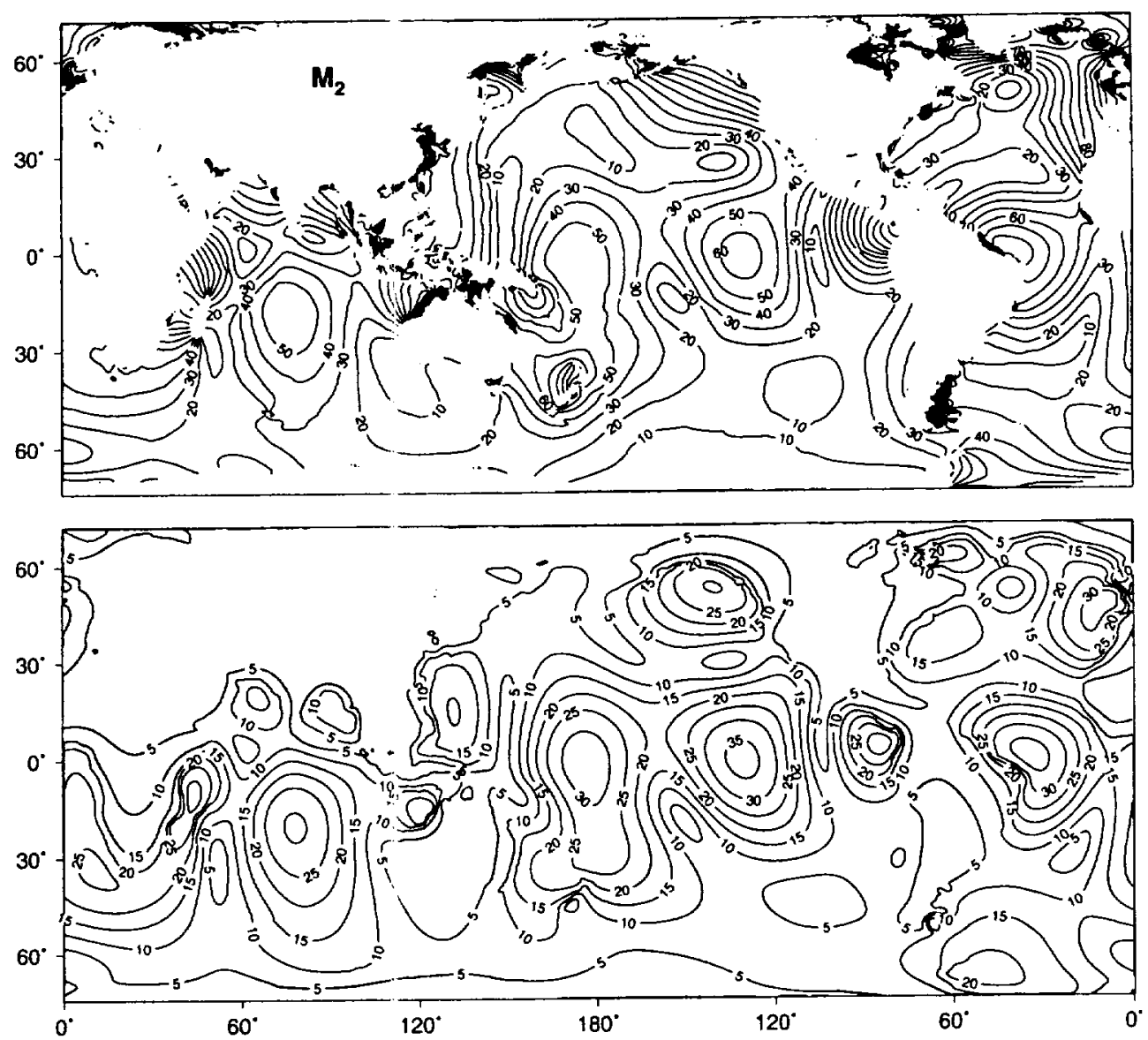

Figure 1. Amplitude of the $\mathrm{M}_{2}$ ocean tide (top) in $\mathrm{cm}$ and the corresponding induced geoid perturbation (bottom) in mm. The ocean model is based primarily on Topex/Poseidon data. The geoid perturbation follows from Eq. (3)

For reference the tidal perturbation in the dimensionless Stokes coefficients $C_{n m}$ are related to the elevation coefficients by

$$
\delta C_{n m}(t)=\frac{3 \rho_{w}}{a \rho_{e}} \frac{\left(1+k_{n}^{\prime}\right)}{(2 n+1)} z_{n m}(t)
$$

At radius $r=a$ the tidal perturbations in the gravity field $-\partial U / \partial r$ are

$$
\delta g(\theta, \varphi, t)=4 \pi G \rho_{w} \sum_{n, m}\left(1+k_{n}^{\prime}\right)\left(\frac{n+1}{2 n+1}\right) z_{n m}(t) Y_{n}^{m}(\theta, \varphi) .
$$

For sufficiently large $n$ the gravity perturbation is related to the tidal height simply by

$$
\lim _{n \rightarrow \infty} \delta g_{n m}=2 \pi G \rho_{w} z_{n m}=(0.0434 \mathrm{mGal} / \mathrm{m}) z_{n m},
$$


which leads to the Bouguer plate approximation $(\delta g \approx 0.0434 \zeta)$ used by Knudsen and Andersen (2002). The approximation works well except for very small $n$.

As Figure 1 shows, the tides perturb the geoid by amounts well below $5 \mathrm{~cm}$. The largest perturbation (fo: $\mathrm{M}_{2}$ ) is $3.7 \mathrm{~cm}$ in the equatorial eastern Pacific. Geoid signals of this magnitude are about an order of magnitude smaller than present uncertainties in the geoid over the ocean (Lemoine et al., 1998), but they are well within the anticipated precisions of GRACE and GOCE (for GOCE, see ESA [1999] Table 8.4).

\subsection{OCEAN TIDE Signai S IN GRACE AND GOCE}

The gravitational signals of ocean tides relevant to the GRACE and GOCE measurements can be predicted with a global ocean tide model. Cheng (2002) recently employed linear perturbation methods to compute the anticipated spectrum of tidal signals in GRACE data over a frequency range of 0-150 cycles/day. We here show an example in the time domain based on a numerical integration of GRACE ephemerides (including initial state adjustments) at altitude approximately 500 $\mathrm{km}$. The relevant forcing from ocean tides is computed using the the four largest constituents of the GOT00.2 ocean model (an update to Ray [1999]) complete to spherical harmonic degree 60 .

Figure 2a shows the expected ocean tide signal in GRACE's range-rate measurement for an arbitrary 120-minute arc segment, or about 1.5 revolutions. Most of this signal should be observable with GRACE's $1-\mu \mathrm{m} / \mathrm{s}$ precision, especially given that these signals are temporally coherent.

As an alternative to employing numerical integration or linear perturbation methods, it is sometimes useful to approximate these range rate signals by employing the following simplistic melhod. For a perfectly circular and polar orbit, the alongtrack acceleration is given by $\hat{\theta} \cdot \nabla U$ and the velocity thus by $(1 / r) \int(\partial U / \partial \theta) d t$. Within the integrand we approximate $\dot{\theta}$ as simply $\left(G M / r^{3}\right)^{1 / 2}$, with $M$ the Earth's mass, so that the along-track velocity integrates to $v(t) \approx(r / G M)^{1 / 2} U(t)$. The range rate $\dot{\rho}$ is then taken as the difference $v(t)-v(t+\Delta t)$, with $\Delta t$ the time lag between the two satellites, ur

$$
\dot{\rho}(t) \approx\left(G M / r^{3}\right)^{1 / 2}(U(t)-U(t+\Delta t) .
$$

Of course, this simple approximation fails to account for the dynamically resonant terms in range rate at 0,1 , and 2 cycles/rev; these terms, which in fact are dominant for GRACE (Cheng, 2002) can only be recovered by integration of the equations of motion. Nonetheless, the method usefully captures the shorter period signals in range-rate, as can be seen in Figure $2 b$. The difference between Figure $2 a$ and $2 b$ is predominantly a sum of two sinusoids of frequency 1 and 2 cycles/rev.

The planned GOCE satellite will measure directly the nine components of the second-order gravity gradient tensor. Figure 3 shows the predicted tidal signal in the second-order radial gradient, which is generally the largest component (Schrama, 


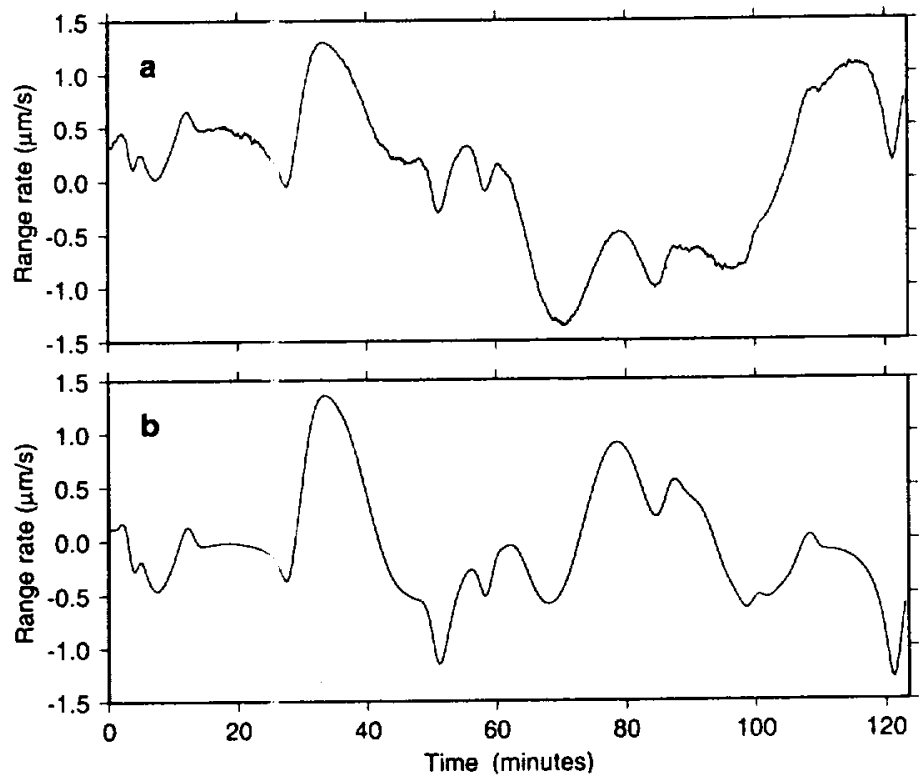

Figure 2. Predicted ocean tide signals in the GRACE range rate measurements for an arbitrary 120 minutes of a 1-day arc, computed (a) by numerical integration with a complete dynamical force model and (b) by the approximation in Eq. (6). The difference between these curves is caused primarily by 1 - and 2-cycle/rev re:tonant terms, not accounted for in (6).

1996). The arc is the same 120-minute arc used above, but artificially lowered to $250-\mathrm{km}$ altitude. The signal is computed simply as the gradient of the potential $U$ in (2). As should be expected, a gradiometer is sensitive to relatively short wavelength components of the tidal mass field. In fact, the signal in Figure 3 tends somewhat to

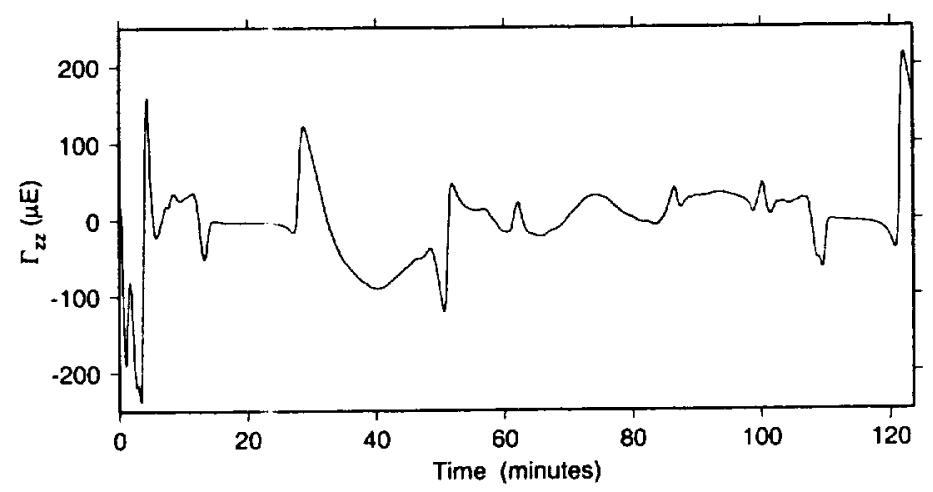

Figure 3. Predicted ocean tide signals in the GOCE gradiometer measurements of $\partial^{2} U / \partial z^{2}$ where $U$ is given by Eq. (2), taken to degree and order 150 for the four largest tidal constituents. The arc is identical to that used for Figure 2 except artificially lowered to $250 \mathrm{~km}$ altitude. Vertical axis units are $\mu \mathrm{E}$, or $10^{-15} \mathrm{~s}^{-2}$ 
mimic the tidal elevation fitld directly below the satellite. For example, the sharp jump near $t=25 \mathrm{~m}$ occurs as the satellite path passes from land to a coastal region of large tide. Unlike the case for GRACE, the tidal signals in GOCE are more than an order of magnitude smaller than the instrument measurement precision, which for the gradiometer is a few $\mathrm{mE} / \mathrm{Hz}^{1 / 2}$ (where 1 Eötvös $=10^{-9} \mathrm{~s}^{-2}$ ). Within the gradiometer measurement band of $0.005-0.1 \mathrm{~Hz}$ (ESA, 1999), tidal signals are likely to be detectable only by exploiting their temporal coherence. GPS tracking of GOCE strengthens the low-frequency measurement precision substantially, which further strengthens the tidal sensitivity of the system (see error spectrum below).

We turn now to a discussion of how well these tidal signals can be modeled as an a priori correction to the satellite measurements.

\section{Errors in Present-Day Tide Models}

Comparisons of models with in situ tide gauge estimates suggest that open ocean tides are now known to better than $2 \mathrm{~cm}$ rms (e.g., Shum et al., 1997; Ray, 1999). Errors in coastal regions, where tides have large amplitudes and high wavenumbers, are probably an order of magnitude worse. Errors are also substantially larger in polar regions above the $\pm 66^{\circ}$ latitude limits of Topex/Poseidon data. We require here a more definite error model, and we consider two types. First, we simply difference some of the better global tide models now available. Since these models were all developed from Topex/Poseidon measurements, the resulting error model may be somewhat optimistic. Secondly, we adopt the global inverse error estimates described by Egbert and Erofeeva (2002). These are based on a Monte Carlo method that is somewhat dependent on assumed prior error covariances for the altimeter data and for the dynamical tidal equations to which the altimeter data are fit. Employing both types of error models is therefore a useful test of consistency. We concentrate on the $\mathrm{M}_{2}$ constituent; errors in other tidal constituents are generally smaller, with the possible exception of $S_{2}$ errors in low latitudes where contamination from the $S_{2}$ atmospheric tide is apparent in some tide models at the level of $1 \mathrm{~cm}$. The error models are described in more detail in Ray et al. (2001).

Figure 4 shows the geoid amplitude spectrum of the $\mathrm{M}_{2}$ tide and our two different error estimates. These spectra are computed from the complex Stokes coefficients $\delta C_{n m}(t)$ (or from their estimated errors) following Eq. (4). The degree amplitude spectrum in the geoid is

$$
\delta N_{n}=a\left(\sum_{m=-n}^{n}\left|\overline{\delta C}_{n m}\right|^{2}\right)^{1 / 2},
$$

where the overbar denotes the rms over a tidal cycle $\left(12.4 \mathrm{~h}\right.$ for $\left.\mathrm{M}_{2}\right)$ in each complex component of $\delta C_{n m}(t)$ This form of $\delta N_{n}$ is consistent with Eq. (4) of Ray et al. (2001) and is also consistent with the geoid spectra of Wahr et al. (1998). 


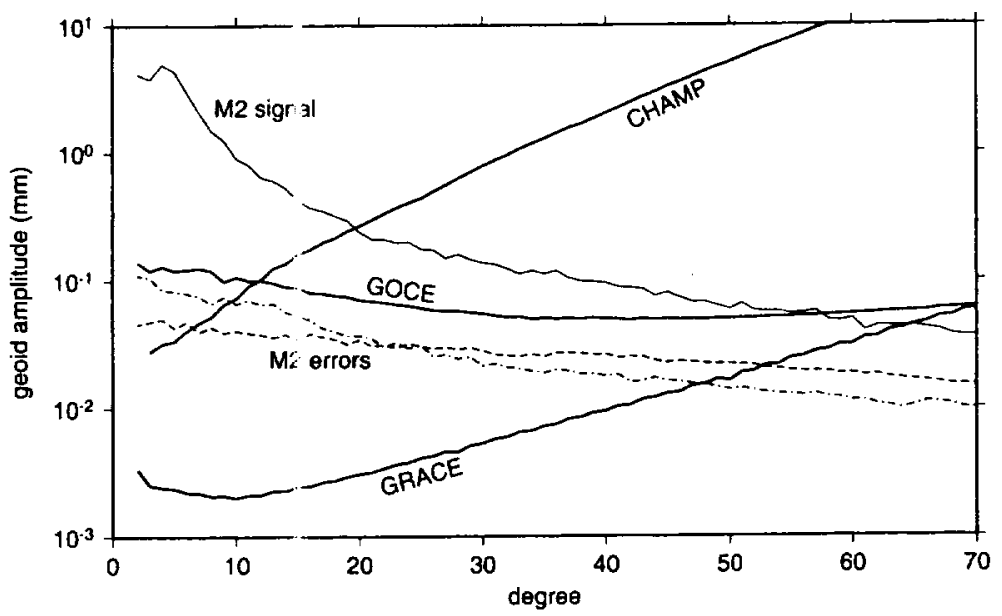

Figure 4. Degree amplitude geoid spectrum of the $\mathrm{M}_{2}$ tide (thin solid line) and two estimates of its error (thin broken lines, the inverse model being the lower curve at low degree). These curves represent rms spectra over a single tidil cycle $(12.4 \mathrm{~h})$. Heavy solid lines denote anticipated sensitivities of three multi-year space geodetic missions, as estimated by Visser et al. (2002). Tidal constituents other than $\mathrm{M}_{2}$ would fall below these $\mathrm{M}_{2}$ curves.

In Figure 4 the $\mathrm{M}_{2}$ error curves are the broken lines, one being the Monte Carlo inverse model, the other being the mean of the differences among models TPXO.5, GOT00.2, and NAO99 (the latter from Matsumoto et al., 2000). The inverse error is the lower curve at small degree $n$ and the higher at large $n$. The two error curves are reasonably consistent and generally within a factor of 2 . The bold lines in Figure 4 represent the sensitivities of three different space geodetic missions after multiple years of data. As expected, the $\mathrm{M}_{2}$ signal is well above the sensitivities of all three satellite missions, which suggests that high-degree tidal models, perhaps to at least degree 70, are required for GRACE and GOCE. Figure 4 also shows that present-day $\mathrm{M}_{2}$ errors exceed the GRACE sensitivity below degree 50 or so. The errors also exceed the CHAMP sensitivity below degree 8 or 10. They do not, however, exceed the expected GOCE sensitivity anywhere. This, and the fact that tidal errors are even smaller than the already very small gradient signals shown in Figure 3, suggests that ide model errors are unlikely to be a major concern for the GOCE mission. It is possible that some large tidal errors in a few coastal regions might degrade GOCE analyses, but such errors are far more problematic for satellite altimetry than for satellite gradiometry.

While Figure 4 suggests that tide models must be improved for (or by) the GRACE and CHAMP missions, the temporal mismatch between the tidal and the satellite curves ( $12.4 \mathrm{~h}$ vs. several years) complicates matters. The manifestation of tidal error in GRACE, for example, depends on issues of sampling and on the desired estimation timescales (e.g., instantaneous gravity anomalies, monthly means, or multi-year means). 


\section{Tidal Errors and GRACE}

The effects of tide model errors on GRACE's intersatellite measurements are sufficiently complex that they are difficult to predict without extensive simulations. We show one example below. Nonetheless, some insight can likely be gained from simple considerations of tidal aliasing. GRACE signals such as those in Figure $2 \mathrm{~b}$ will be aliased in ways quite similar to the aliasing in satellite altimetry. One must bear in mind, however, that the spectrum of tide-induced signals in GRACE is richer than Figure 2b suggests (e.g., Cheng, 2002). GRACE is no altimeter, and some of its tidal errors are entangled with orbit errors with potentially complicated aliasing.

\subsection{Tidal Aliasing}

The tidal phases observed by an orbiting spacecraft are determined by the angle between the satellite orbit plane and the tide-raising body. The orbit plane of any high-inclination spacecraft like GRACE precesses so slowly with respect to the sun that solar tides are necessarily aliased into relatively long periods-e.g., semiannual, annual, or longer. Such periods are of prime importance to climatological studies, making aliased errors at these periods very problematic. (The precession rate with respect to the moon is rapid, and aliasing of lunar tides is rarely problematic unless one happens to be unlucky.) Because the solar tides that GRACE observes will be aliased to long periods, we may predict that errors will be prevalent in the GRACE measurements at these same periods, no matter the exact manner in which the errors are manifested through dynamical complications in the orbit.

From the satellite precession rate we may determine the tidal alias periods. The alias period of the $S_{1}$ tide is identical to the satellite nodal period with respect to the mean sun; the $S_{2}$ period is half that. The alias period of $K_{1}$ is identical to the satellite nodal period with respect to the mean equinox; $K_{2}$ is half that. The $P_{1}$ alias is identical to the nodal period with respect to a fictitious body moving at twice the sun's mean motion. As of this writing (a few months after launch) the GRACE mean Keplerian elements are $(a, e, l)=\left(6860.4 \mathrm{~km}, 0.00256,89.02^{\circ}\right)$, implying a node rate of approximately $-0.132^{\circ} /$ day, or $-1.117^{\circ} /$ day with respect to the mean sun. The corresponding alias periods for the primary solar tides are:

$\begin{array}{llll}\mathrm{K}_{2} & 3.74 \text { years } & \mathrm{K}_{1} & 7.48 \text { years } \\ \mathrm{S}_{2} & 161 \text { days } & \mathrm{S}_{1} & 322 \text { days } \\ \mathrm{P}_{1} & 171 \text { days } & & \end{array}$

Some tidal contamination should therefore be expected at the important semiannual period and at the interannual periods of 3.7 and 7.5 years. The $S_{1}$ alias is also somewhat problematic, since both $S_{1}$ (being primarily atmospheric) and the nearby annual cycle (Sa) have strong temporal variability, causing potential overlap of their rather broad spectral peaks. 


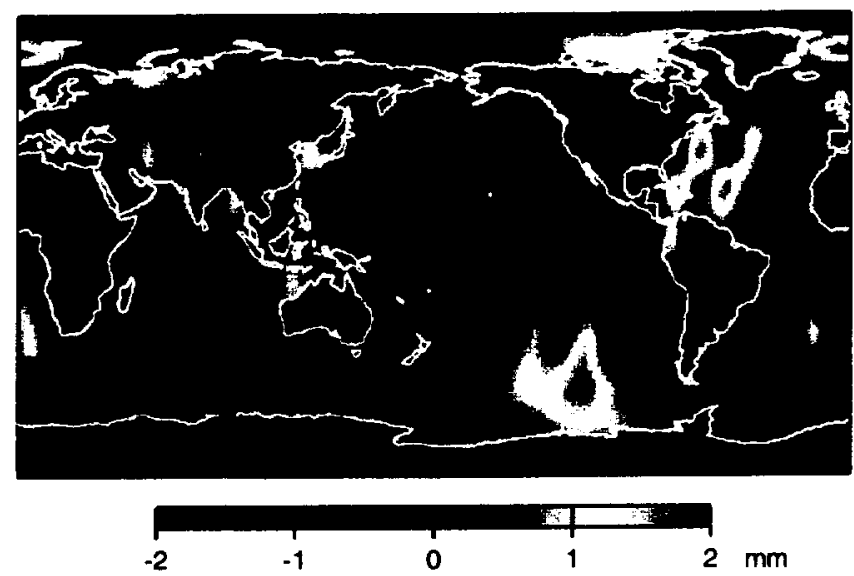

Figure 5. Results of GRACE simulation showing errors in an estimated monthly geoid (in mm) caused by tide model errors. Gevid is evaluated to degree and order 22; higher degree expansions show larger tidal contamination. Note errors are not confined to the oceans, primarily because of the long-period dynamical perturbations to GRACE's range rate.

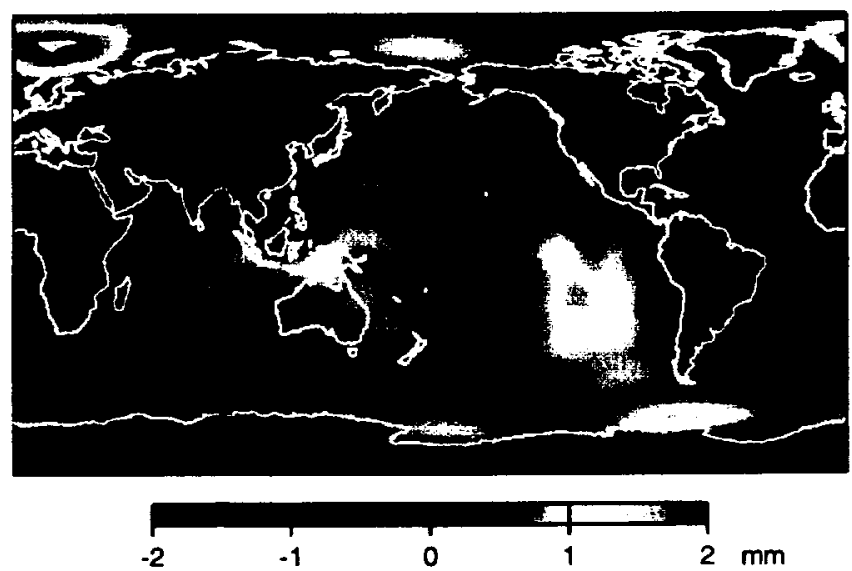

Figure 6. As in Figure 5 except $\mathrm{using}$ the simplistic (non-dynamic) approach of Eq. (6). Errors are more localized to the ocean.

\subsection{Simulation of Monthly Gravity ERrors}

Because the GRACE project intends to generate monthly mean estimates of the geopotential (Tapley and Reigber, 2000), it is of interest to anticipate possible contamination of this product caused by errors in the tide model adopted for processing the GRACE measurements. We have estimated this effect by simulating one (arbitrarily chosen) month of GRACE range-rate measurements, using the methods described by Rowlands et al. (2002). The simulation consists of an inversion for a degree-120 static geopotential in the presence of realistic errors in intersatellite 
range measurements and in GPS tracking, with and without tide model errors. The latter consists of the difference between tide models GOT00.2 and TPXO.5, to degree 60 , for constituents $\mathrm{M}_{2}, \mathrm{~S}_{2}, \mathrm{~N}_{2}, \mathrm{~K}_{2}, \mathrm{O}_{1}$ and $\mathrm{K}_{1}$. The resulting tidal contamination in the monthly mean geoid estimate is shown in Figure 5. Because errors in monthly geoid estimates rise rapidly with degree, the geoid in Figure 5 is evaluated only to degree 22 . Summing to degree 50 results in fairly similar characteristics, although with more orbit stripping and roughly double amplitudes.

The simulation represented by Figure 5 is computationally expensive, and it is therefore of interest to see how well the simplistic (and inexpensive) formula (6) can represent the same error field (akin to comparing Figures $2 a$ and $2 b$ ). This involves no ephemeris computations and only a relatively small normal matrix. The result is shown in Figure 6. Because this method ignores the dynamical long-period effects, there is no track-type stripping and errors are confined primarily to ocean regions. The errors in both figures are of comparable magnitude and they align in a few locations (e.g., Amazon mouth, Weddell Sea), but the method underlying Figure 6 is evidently too simplistic to map the error in detail.

Several points about Figure 5 are worth noting. The presence of geoid errors over both land and ocean is caused by the rich spectrum that tidal forcing induces in the range-rate measurements. Localized errors in ocean tide models can induce global errors in gravity estimation. Secondly, the amplitude of these geoid errors is significant for some anticipated GRACE applications. In particular, any interpretation of the estimated gravity in terms of oceanic mass motions is considerably confounded. Wahr et al. (1998; their Plate 1) estimate annual variations in the geoid of about $1 \mathrm{~mm}$ and less, based on a general circulation model, and such signals are comparable to the errors in Figure 5. Thirdly, the errors in Figure 5 are one monthly manifestation and we have no clue to their temporal variations without extending the simulations over a considerably longer timespan. From our discussion of aliasing, however, we anticipate that some of these errors have periods of semi-annual and longer. The $S_{2}$ and $K_{2}$ tides are likely to be most problematic for monthly mean gravity estimates (Knudsen and Andersen, 2002).

\section{Summary Discussion}

The ocean tides induce variations in the geoid of several $\mathrm{cm}$, well within the anticipated precisions of the latest space geodetic missions. Yet it is important to realize that the geoid errors in present-day gravity models are a few decimeters over the ocean (Lemoine et al., 1998), whereas errors in the prediction of tidal geoid variations are orders of magnitude smaller. We conclude that missions like GRACE and GOCE can inıprove our knowledge of the geopotential by an order of magnitude, or more, even with present ocean tide models. Tide model errors become crucial with the extreme precisions required for GRACE to detect temporal gravity changes of the sort discussed by Wahr et al. (1998). 
To the extent that Figure 4 is realistic, GRACE itself offers opportunities to improve the long wavelength components of ocean tide models, specifically coefficients at degrees 50 and below, or wavelengths of about $1000 \mathrm{~km}$ and longer. CHAMP may also help improve a few low-degree coefficients. These improvements are likely to occur only after multi-year time series have been obtained, because many tides are aliaked to nearby frequencies. The solar tides will be especially problematic. For example, GRACE will not sample a complete phase cycle of $\mathrm{K}_{1}$ until nearly four years have passed, and such long periods will contain substantial power from non-tidal signals. The aliasing of certain tides into semiannual and near-annual periods will also make solar tide improvements difficult. Lunar tides will be less problematic, and improvements even to $\mathrm{M}_{2}$ will be most welcome.

Space limitations have forced us to concentrate on the subject of tides as a correction to new satellite gravimetry missions. But if history is a guide, then the markedly enhanced precisions of these new measurements will also yield new information about the tides - solid and atmospheric as well as oceanic-and such improvements can in turn yield further information about other aspects of the Earth system. As just one example, improvements in degree- 2 and degree- 3 tides shed light on the Earth's anelasticity at frequencies well outside the seismic band. Determination of these low-degree coefficients should improve significantly with the new satellite data.

\section{Acknowledgements}

We thank Nikos Pavlis, Ernst Schrama, and John Wahr for useful discussions. Pieter Visser kindly provided the three satellite error curves shown in Figure 4.

\section{References}

Cheng, M. K.: 2002, 'Gravitation al perturbation theory for intersatellite tracking', J. Geod. 76, 169185.

Egbert, G. D. and S. Y. Erofeev: : 2002, 'Efficient inverse modeling of barotropic ocean tides', $J$. Atmos. Oceanic Tech. 19, 183-204.

ESA: 1999, Gravity Field and Steady-State Ocean Circulation Mission, Rep. SP-1233, European Space Agency, Noordwijk, 217 pp.

Knudsen, P. and O. Andersen: 2002, 'Correcting GRACE gravity fields for ocean tide effects', Geophys. Res. Lett. 29 (8), 19-1-19-4.

Lambeck, K.: 1988, Geophysical Geodesy, Clarendon Press, Oxford.

Lemoine, F. et al.: 1998, The development of the joint NASA GSFC and NIMA geopotential model EGM96, NASA Tech. Memo 206861, Goddard Space Flight Center, Greenbelt, 575 pp.

Matsumoto, K., T. Takanezawa. M. Ooe: 2000, 'Ocean tide models developed by assimilating Topex/Poseidon altimeter dat 1 into hydrodynamical model', J. Oceanogr. 56, 567-581.

Pavlis, E. C. and L. Iorio: 2002, 'The impact of tidal errors on the determination of the Lense-Thirring effect from satellite laser ranging', Int. J. Mod. Phys. D, 11, 599-618. 
Ray, R. D.: 1999, A global ocean tide model from Topex/Poseidon altimetry, NASA Tech. Memo. 209478, Goddard Space Flight Center, Greenbelt, 58 pp.

Ray, R. D., R. J. Eanes, G. D. Egłert, N. K. Pavlis: 2001, 'Error spectrum for the global $\mathrm{M}_{2}$ ocean tide', Geophys. Res. Lett. 28, 21-24.

Rowlands, D. D., R. D. Ray, D. S. Chinn, F. G. Lemoine: 2002, 'Short-arc analysis of intersatellite tracking data in a gravity mapping mission', J. Geod. in press.

Schrama, E. J. O.: 1996, 'Gravity research missions reviewed in light of the indirect ocean tide potential', in R. H. Rapp, A. A. Cazenave and R. S. Nerem (eds.), Global Gravity Field and lts Temporal Variations, Springer, Berlin, pp. 131-140.

Shum, C. K. et al.: 1997, 'Accuritcy assessments of recent global ocean tide models', J. Geophys. Res. 102, 25173-25194.

Tapley, B. D. and C. Reigber: 2000, 'The GRACE mission: status and future plans' (abstract), EOS, Trans. AGU, 81, Fall Suppl., F307.

Visser, P. et al.: 2000, 'The Europian Earth Explorer Mission GOCE: Impact for the geosciences', in J. Mitrovica and L. Vermeersen (eds.), Ice Sheets, Sea Level and the Dynamic Earth, American Geophysical Union, Washington, in press.

Wahr, J., M. Molenaar, F. Bryan: 1998. 'Time variability of the Earth's gravity field: hydrological and oceanic effects and their possible detection using GRACE', J. Geophys. Res. 103, 30205-30229.

Address for Offprints: R. D. Ray, NASA GSFC, Code 926, Greenbelt MD 20771, USA richard.ray@gsfc.nasa.gov 